\title{
Publisher's Note: Large Deviation Function for the Number of Eigenvalues of Sparse Random Graphs inside an Interval [Phys. Rev. Lett. 117, 104101 (2016)]
}

Fernando L. Metz and Isaac Pérez Castillo

(Q) (Received 30 October 2020; published 18 November 2020)

DOI: 10.1103/PhysRevLett.125.219901

This Letter was published online on 1 September 2016 with an improperly formatted author affiliation. Isaac Pérez Castillo's affiliation should read "Departamento de Sistemas Complejos, Instituto de Física, Universidad Nacional Autónoma de México, Cd. de México C.P. 04510, México". The Letter has been corrected as of 9 November 2020. The author affiliation is incorrect in the printed version of the journal. 